\title{
Superconducting Fluctuation and Pseudogap in Disordered Short Coherence Length Superconductor
}

\author{
Youichi YANASE* \\ Department of Physics, University of Tokyo, Tokyo 113-0033, Japan \\ Theoretische Physik, ETH-Honggerberg, 8093 Zurich, Switzerland
}

(Received August 17, 2006)

\begin{abstract}
We investigate the role of disorder on the superconducting (SC) fluctuation in short coherence length $d$-wave superconductors. The particular intetest is focused on the disorder-induced microscopic inhomogeneity of SC fluctuation and its effect on the pseudogap phenomena. We formulate the self-consistent 1-loop order theory for the SC fluctuation in inhomogeneous systems and analyze the disordered $t-t^{\prime}-V$ model. The SC correlation function, electronic DOS and the critical temperature are estimated. The SC fluctuation is localized like a nanoscale granular structure when the coherence length is short, namely the transition temperature is high. This is contrasted to the long coherence length superconductors where the order parameter is almost uniform in the microscopic scale. In the former case, the SC fluctuation is enhanced by the disorder in contrast to the Abrikosov-Gorkov theory. These results are consistent with the STM, NMR and transport measurements in high- $T_{\mathrm{c}}$ cuprates and illuminate the essential role of the microscopic inhomogeneity. We calculate the spacial dependence of DOS around the single impurity and discuss the consistency with the NMR measurements.
\end{abstract}

KEYWORDS: Microscopic inhomogeneity; pseudogap; superconducting fluctuation; high- $\mathrm{T}_{\mathrm{c}}$ cuprates; randomness

\section{Introduction}

The pseudogap phenomena in high- $T_{\mathrm{c}}$ cuprates have been one of the central issues in strongly correlated electron systems. ${ }^{1}$ The theoretical elucidation of anomalous properties is highly desired for a comprehensive understanding of high- $T_{\mathrm{c}}$ superconductivity.

Among many theoretical proposals, the "pairing scenario" is one of the promising ideas. Then, the origin of pseudogap is attributed to the superconducting correlation above $T_{\mathrm{c}} \cdot{ }^{2-14} \mathrm{In}$ contrast to the conventional superconductors, the coherence length is extraordinary short in under-doped cuprates, which is typically a few lattice spacing. The superconducting (SC) fluctuation is enhanced in such a short coherence length superconductor with quasi-2D lattice structure.

In order to examine the validity of pairing scenario it is important to investigate the roles of disorder and/or magnetic field which suppress the $d$-wave superconductivity. As for the magnetic field, it has been shown that the pseudogap induced by the SC fluctuation is slightly affected by the orbital de-pairing effect around the onset temperature $T=T^{*} \cdot{ }^{15}$ This is mainly because the correlation length of superconductivity is still short at $T=T^{*}$, where the pseudogap is induced by the short range SC correlation. On the other hand, the critical fluctuation around $T=T_{\mathrm{c}}$ is remarkably affected by the magnetic field because the correlation length diverges at the critical point. ${ }^{15,16}$ These results are consistent with NMR measurements. ${ }^{17,18}$

In this paper, we investigate the role of disorder on the short coherence length superconductor with $d$-wave symmetry. This issue is particularly interesting because the microscopic inhomogeneity observed in recent STM measurements ${ }^{19-21}$ indicates a novel effect of disorder in the under-doped region. Furthermore, it is considered that the disorder plays an essential role in the superconductorinsulator (SI) transition in high- $T_{\mathrm{c}}$ cuprates as implied by the disordered magnetism in the LSCO and YBCO compounds. ${ }^{22-26}$

The pseudogap induced by the $d$-wave SC fluctuation has been investigated in disordered system on the basis of the self-consistent t-matrix approximation (SCTMA) ${ }^{27}$ for the disorder. ${ }^{28,29}$ Then, it was shown that the pseudogap and SC fluctuation are significantly suppressed by the disorder. This conclusion seems to be reasonable since the $T_{\mathrm{c}}$ in non- $s$-wave superconductors is suppressed by the disorder. Then, the thermal fluctuation can be suppressed. However, this result is incompatible with the experimental results which have indicated a robustness of pseudogap in disorder-doped materials. ${ }^{30-32}$

In this paper, we carry out the calculation beyond the SCTMA where the disorder is exactly taken into account. Contrary to the SCTMA, it is shown that the SC fluctuation is enhanced by the microscopic inhomogeneity which is characteristic in the quasi-2D short coherence length superconductor with non-s-wave symmetry. Then, the long range coherence hardly develops owing to the nanoscale inhomogeneity of SC order parameter while the well developed short range correlation leads to the pseudogap in the low energy spectrum. This result illuminates the breakdown of Abrikosov-Gorkov theory for the short coherence length superconductors.

We analyze the $t-t^{\prime}-V$ model to investigate the microscopic role of disorders on the SC fluctuation. We adopt the 1-loop order approximation with respect to the SC fluctuation, which is called "self-consistent Tmatrix approximation"..$^{2-4,27}$ This approach is complementary with the phase fluctuation theory ${ }^{6-13}$ which is 
a phenomenological description for the deeply critical region. It has been shown that the 1-loop order theory is consistent with many experimental results in the pseudogap state. The anomalous behaviors in single particle, magnetic and transport properties ${ }^{1,33}$ are explained in a comprehensive way by taking into account the spin fluctuation in addition to the SC fluctuation. ${ }^{14,34,35}$ Furthermore, the microscopic estimation of the SC fluctuation in Hubbard model is consistent with the remarkable electron-hole asymmetry in high- $T_{\mathrm{c}}$ cuprates. ${ }^{34}$

This paper is organized as follows. The disordered $t$ $t^{\prime}-V$ model is introduced in $\S 2$, where we formulate the self-consistent T-matrix approximation ${ }^{27}$ in disordered systems. In $\S 3.1$, the typical results on the spatial dependence of SC fluctuation are shown. In $\S 3.2$, we numerically take the random average and calculate the DOS, $\mathrm{SC}$ correlation function and $T_{\mathrm{c}}$. The role of SC fluctuation is clarified by comparing with the mean field theory. The effects of microscopic inhomogeneity are illuminated by the comparison with the SCTMA for disorder effects. In $\S 4$, we briefly discuss the spatial dependence of DOS around the single impurity. Some discussions are given in the last section $\S 5$.

\section{Formulation in the Disordered $t-t^{\prime}-V$ Model}

\subsection{1-loop order theory in disordered systems}

We adopt the disordered $t-t^{\prime}-V$ model which is expressed as,

$$
\begin{gathered}
H=-t \sum_{<i, j>, \sigma} c_{i \sigma}^{\dagger} c_{j \sigma}+t^{\prime} \sum_{\ll i, j \gg, \sigma} c_{i \sigma}^{\dagger} c_{j \sigma} \\
+\frac{V}{2} \sum_{<i, j>} n_{i} n_{j}+\sum_{i}\left(W_{i}-\mu\right) n_{i},
\end{gathered}
$$

where $n_{i}$ is the electron number at the site $i$. The symbols $\langle i, j\rangle$ and $\ll i, j \gg$ denote the summation over the nearest neighbor sites and that over the next nearest neighbor sites, respectively. The attractive interaction $V<0$ between the nearest neighbor sites favors the $d_{\mathrm{x}^{2}-\mathrm{y}^{2}}$-wave superconductivity around the half filling. The disorder is introduced by the disordered potential $W_{i}$. Although we have investigated several kinds of disorder including the box disorder, Gaussian disorder and $t^{\prime}$-disorder, we show the results for the point disorder where $W_{i}=0$ or $W_{i}=W$. Since many experimental studies on the Zn-doped cuprates have been reported, we assume the strong disorder $W=40 t \gg t$. Then, the sites $i$ where $W_{i}=W$ correspond to the Zn sites and the others are the $\mathrm{Cu}$ sites. Recently, the disorder outside the plane has attracted much interests. ${ }^{20,21,36}$ Then, the disordered potential is described by the extended point disorder. We have confirmed that the following results are qualitatively independent of the type of disorder.

It should be noted that the $t-t^{\prime}-V$ model is a semiphenomenological model for the short coherence length $d$-wave superconductor. The $d$-wave pairing in high- $T_{\mathrm{c}}$ cuprates is originally induced by the short range repulsive interaction through the many body effect. We have analyzed the repulsive Hubbard model within the FLEX+T-matrix approximation, ${ }^{34}$ and shown that the attractive models like eq. (1) appropriately capture the role of SC fluctuation. ${ }^{14}$ In this paper, the effect of the microscopic inhomogeneity will be elucidated from the general point of view.

We choose the unit of energy as $t=1$ and fix $t^{\prime} / t=$ 0.25 . The chemical potential $\mu$ is fixed to $\mu=-0.8$ which corresponds to the $10 \%$ hole doping. The typical Fermi surface of under-doped cuprates is well reproduced by this parameter set. Since we fix the chemical potential instead of the total electron number, the average electron density per $\mathrm{Cu}$ site $n=\sum_{<i>} n_{i} / N_{0}$ is little affected by the disorder-doping. Here, $\sum_{<i>}$ denotes the summation over the sites where $W_{\mathrm{i}}=0 . N_{0}$ is the number of those sites. Although the effect of impurity doping on the average electron density in high- $T_{\mathrm{c}}$ cuprates is not clear, we can study the general roles of disorder by analyzing this model.

We define the non-interacting part of Hamiltonian as follows,

$$
\begin{aligned}
H_{0}= & -t \sum_{<i, j>, \sigma} c_{i \sigma}^{\dagger} c_{j \sigma}+t^{\prime} \sum_{<<i, j>>, \sigma} c_{i \sigma}^{\dagger} c_{j \sigma}+\sum_{i}\left(W_{i}-\mu\right) n_{i} \\
& =\sum_{\sigma} \hat{c}_{\sigma}^{\dagger} \hat{H}_{0} \hat{c}_{\sigma}
\end{aligned}
$$

Here, $\hat{c}_{\sigma}^{\dagger}=\left(c_{i, \sigma}^{\dagger}\right)$ is the $N$-component vector and $N=$ $L \times L$ is the total number of sites. The Green function in the non-interacting system $(V=0)$ is obtained by diagonalizing the $N \times N$ Hermitian matrix $\hat{H}_{0}$ as,

$$
G_{0}\left(\hat{i}, \hat{j}, \omega_{n}\right)=\sum_{l} u(m, l) \frac{1}{\mathrm{i} \omega_{n}-\varepsilon_{l}} u^{*}(n, l),
$$

where $\varepsilon_{l}$ is the $l$-th eigenvalue of the matrix $\hat{H}_{0}$ and $u(m, l)$ is the $m$-th element of the eigenvector. The index $m(n)$ is defined so that the $m$-th $(n$-th) element of $\hat{c}_{\sigma}^{\dagger}$ is the creation operator at site $\hat{i}(\hat{j})$. The fermion Matsubara frequency is given as $\omega_{n}=(2 n+1) \pi T$. Since we have included the disorder potential into the unperturbed Hamiltonian, the disorder is exactly taken into account in the following calculation.

In case of $V \neq 0$, the dressed Green function is obtained by the Dyson equation as,

$$
\begin{aligned}
G\left(\hat{i}, \hat{j}, \omega_{n}\right)= & G_{0}\left(\hat{i}, \hat{j}, \omega_{n}\right)+ \\
& \sum_{\hat{i}^{\prime}, \hat{j}^{\prime}} G_{0}\left(\hat{i}, \hat{i}^{\prime}, \omega_{n}\right) \Sigma\left(\hat{i}^{\prime}, \hat{j}^{\prime}, \omega_{n}\right) G\left(\hat{j}^{\prime}, \hat{j}, \omega_{n}\right),
\end{aligned}
$$

where $\Sigma\left(\hat{i}, \hat{j}, \omega_{n}\right)$ is the self-energy. In this paper we estimate the self-energy within the self-consistent or nonself-consistent T-matrix approximation whose results in the clean system have been summarized in Ref. 14 .

It generally takes a long computational time to calculate the self-energy in the inhomogeneous system because the Green function $G\left(\hat{i}, \hat{j}, \omega_{n}\right)$ depends not only on the relative coordinate $\hat{i}-\hat{j}$ but also on the center of mass coordinate $(\hat{i}+\hat{j}) / 2$. In order to reduce the computational time, we apply the quasi-static approximation for the propagator of SC fluctuation, namely the T-matrix. The quantum dynamics of SC fluctuation, namely the imaginary time dependence of fluctuation propagator, is ignored in this approximation and only the thermal fluctuation is taken into account. ${ }^{37-46}$ This approximation 
(a)

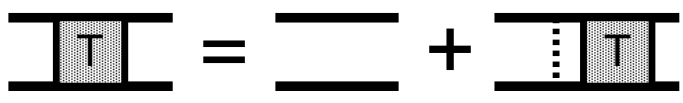

(b)

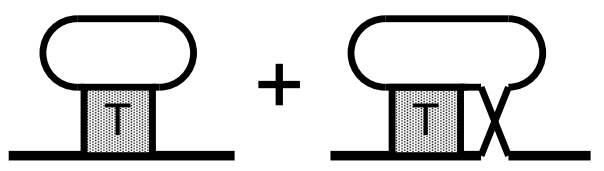

Fig. 1. The diagrammatic representation of (a) T-matrix and (b) self-energy.

is valid at finite temperature around the critical point. We have used the quasi-static approximation for the estimation of higher order corrections beyond the 1-loop order. ${ }^{46}$ Then, we have found that this approximation is quantitatively justified in the pseudogap region. It should be noted that the quasi-static approximation is particularly appropriate for the SC fluctuation owing to the characteristic dynamical property. ${ }^{46}$

Since the T-matrix having non-zero Matsubara frequency $\Omega_{n} \neq 0$ is ignored in the quasi-static approximation, we simply drop the variable of frequency in the T-matrix. Then, the T-matrix is obtained by the following equations,

$$
\begin{aligned}
T\left(\hat{i}, \hat{j}, \hat{\delta}, \hat{\delta}^{\prime}\right)= & T_{0}\left(\hat{i}, \hat{j}, \hat{\delta}, \hat{\delta}^{\prime}\right)- \\
& \sum_{k, \hat{\delta}^{\prime \prime}} T_{0}\left(\hat{i}, \hat{k}, \hat{\delta}, \hat{\delta}^{\prime \prime}\right) V T\left(\hat{k}, \hat{j}, \hat{\delta}^{\prime \prime}, \hat{\delta}^{\prime}\right) \\
T_{0}\left(\hat{i}, \hat{j}, \hat{\delta}, \hat{\delta}^{\prime}\right)= & T \sum_{m} G\left(\hat{i}, \hat{j}, \omega_{m}\right) G\left(\hat{i}+\hat{\delta}, \hat{j}+\hat{\delta}^{\prime},-\omega_{m}\right),(())
\end{aligned}
$$

where $\hat{\delta}=( \pm 1,0)$ or $\hat{\delta}=(0, \pm 1)$. The diagrammatic representation of T-matrix is shown in Fig. 1(a).

The self-energy is represented in Fig. 1(b) and the expression is given as,

$$
\begin{aligned}
\Sigma\left(\hat{i}, \hat{j}, \omega_{n}\right)=-V^{2} \sum_{\delta, \delta^{\prime}} & {\left[T_{\mathrm{s}}\left(\hat{i}, \hat{j}, \hat{\delta}, \hat{\delta}^{\prime}\right)+3 T_{\mathrm{t}}\left(\hat{i}, \hat{j}, \hat{\delta}, \hat{\delta}^{\prime}\right)\right] } \\
\times & \times\left(\hat{j}+\hat{\delta}^{\prime}, \hat{i}+\hat{\delta},-\omega_{n}\right),
\end{aligned}
$$

where

$$
T_{\mathrm{s}, \mathrm{t}}\left(\hat{i}, \hat{j}, \hat{\delta}, \hat{\delta}^{\prime}\right)=\frac{1}{2} T\left(\hat{i}, \hat{j}, \hat{\delta}, \hat{\delta}^{\prime}\right) \pm \frac{1}{2} T\left(\hat{i}, \hat{j}+\hat{\delta}^{\prime}, \hat{\delta},-\hat{\delta}^{\prime}\right) .
$$

Here, $T_{\mathrm{s}}\left(\hat{i}, \hat{j}, \hat{\delta}, \hat{\delta}^{\prime}\right)\left(T_{\mathrm{t}}\left(\hat{i}, \hat{j}, \hat{\delta}, \hat{\delta}^{\prime}\right)\right)$ is the fluctuation propagator for the spin singlet (triplet) pairing. The former includes the $d_{\mathrm{x}^{2}-\mathrm{y}^{2}}$-wave and extended $s$-wave pairings while the latter includes the $p_{\mathrm{x}}$-wave and $p_{\mathrm{y}}$-wave pairings. The coefficient 3 of $T_{\mathrm{t}}\left(\hat{i}, \hat{j}, \hat{\delta}, \hat{\delta}^{\prime}\right)$ in eq. (7) is due to the spin 1 of Cooper pairs in the spin triplet pairing.

Although the $d$-wave pairing state is most stable in the $t-t^{\prime}-V$ model, the pairing correlation develops also in the $p$-wave channel. The latter is expected to be an artifact of the $t-t^{\prime}-V$ model because the $p$-wave pairing instability is negligible in the microscopic model, such as the Hubbard, $d-p$ and $t-J$ models. ${ }^{14,47,48}$ Therefore, we simply drop the contribution from the spin triplet SC fluctuation in eq. (7) and obtain the self-energy as,

$\Sigma\left(\hat{i}, \hat{j}, \omega_{n}\right)=-V^{2} \sum_{\delta, \delta^{\prime}} T_{\mathrm{s}}\left(\hat{i}, \hat{j}, \hat{\delta}, \hat{\delta}^{\prime}\right) G\left(\hat{j}+\hat{\delta}^{\prime}, \hat{i}+\hat{\delta},-\omega_{n}\right) \cdot(9)$

It should be again stressed that the $t-t^{\prime}-V$ model is a semi-phenomenological model which is relevant for the $d$-wave SC fluctuation. Note that the extended $s$-wave pairing fluctuation is also included in $T_{\mathrm{s}}\left(\hat{i}, \hat{j}, \hat{\delta}, \hat{\delta}^{\prime}\right)$ and we cannot separate these pairings in the disordered system owing to the violation of 4 -fold rotational symmetry. We have confirmed that the contribution from the extended $s$-wave pairing is not important.

In the self-consistent T-matrix approximation, the selfenergy, Green function and T-matrix are obtained by solving the self-consistent equations, namely eqs. (3-6) and (8-9). The non-self-consistent T-matrix approximation is given by replacing the Green function $G$ in eqs. (6) and (9) with the unperturbed one $G_{0}$. The self-consistent calculation is carried out for up to $N=31 \times 31$ sites, while the non-self-consistent one is performed for up to $N=65 \times 65$ sites.

\subsection{SCTMA for disorder}

The superconductivity in the disordered alloy has been investigated for more than four decades. ${ }^{49}$ The Born approximation or SCTMA have been used to discuss the macroscopic properties. Then, the random average is taken in advance and the translational symmetry is reestablished in the results. The role of strong disorder on the gap-less superconductivity has been elucidated with use of the SCTMA. ${ }^{50-52}$ However, the role of inhomogeneity is not appropriately taken into account in the SCTMA because the interference effect of many disorders is neglected. In contrast to that, the disorder is exactly taken into account in the formulation in $\S 2.1$. Therefore, it is interesting to illuminate the role of microscopic inhomogeneity by the comparison with SCTMA. In this subsection, we briefly explain the formulation of SCTMA in the $t-t^{\prime}-V$ model.

Since the disorder is taken into account in an approximate way, the unperturbed Hamiltonian is defined as,

$H_{0}^{\prime}=-t \sum_{<i, j>, \sigma} c_{i \sigma}^{\dagger} c_{j \sigma}+t^{\prime} \sum_{<<i, j>>, \sigma} c_{i \sigma}^{\dagger} c_{j \sigma}-\mu \sum_{i, \sigma} n_{i}$.

Then, the Green function, self-energy and T-matrix can be written in the $k$-space representation. For example, the Green function is written as, ${ }^{28,29}$

$$
G\left(\boldsymbol{k}, \omega_{n}\right)=\frac{1}{\mathrm{i} \omega_{n}-\varepsilon(\boldsymbol{k})-\Sigma_{\mathrm{s}}\left(\boldsymbol{k}, \omega_{n}\right)-\Sigma_{\mathrm{d}}\left(\omega_{n}\right)},
$$

where $\varepsilon(\boldsymbol{k})$ is the dispersion relation,

$$
\varepsilon(\boldsymbol{k})=-2 t\left(\cos k_{\mathrm{x}}+\cos k_{\mathrm{y}}\right)+4 t^{\prime} \cos k_{\mathrm{x}} \cos k_{\mathrm{y}}-\mu \text {. }
$$

The self-energy is given by the contribution from the SC fluctuation $\Sigma_{\mathrm{s}}\left(\boldsymbol{k}, \omega_{n}\right)$ and that from the disorder $\Sigma_{\mathrm{d}}\left(\omega_{n}\right)$. In order to compare with the formulation in $\S 2.1$, we calculate the self-energy $\Sigma_{\mathrm{s}}\left(\boldsymbol{k}, \omega_{n}\right)$ in the same approximation as in $\S 2.1$. Then, $\Sigma_{\mathbf{s}}\left(\boldsymbol{k}, \omega_{n}\right)$ is expressed in the real space as,

$$
\Sigma_{\mathrm{s}}\left(\hat{i}, \omega_{n}\right)=-V^{2} \sum_{\delta, \delta^{\prime}} T_{\mathrm{s}}\left(\hat{i}, \hat{\delta}, \hat{\delta}^{\prime}\right) G\left(-\hat{i}-\hat{\delta}+\hat{\delta}^{\prime},-\omega_{n}\right)
$$


where

$$
T_{\mathrm{s}}\left(\hat{i}, \hat{\delta}, \hat{\delta}^{\prime}\right)=\frac{1}{2} T\left(\hat{i}, \hat{\delta}, \hat{\delta}^{\prime}\right)+\frac{1}{2} T\left(\hat{i}-\hat{\delta}^{\prime}, \hat{\delta},-\hat{\delta}^{\prime}\right),
$$

and

$$
\begin{gathered}
T\left(\hat{i}, \hat{\delta}, \hat{\delta}^{\prime}\right)=T_{0}\left(\hat{i}, \hat{\delta}, \hat{\delta}^{\prime}\right)- \\
\sum_{k, \hat{\delta}^{\prime \prime}} T_{0}\left(\hat{i}-\hat{k}, \hat{\delta}, \hat{\delta}^{\prime \prime}\right) V T\left(\hat{k}, \hat{\delta}^{\prime \prime}, \hat{\delta}^{\prime}\right), \\
T_{0}\left(\hat{i}, \hat{\delta}, \hat{\delta}^{\prime}\right)=T \sum_{m} G\left(\hat{i}, \omega_{m}\right) G\left(\hat{i}+\hat{\delta}-\hat{\delta}^{\prime},-\omega_{m}\right) .
\end{gathered}
$$

The $k$-space representations of $\Sigma_{\mathbf{s}}\left(\boldsymbol{k}, \omega_{n}\right)$ and $G\left(\boldsymbol{k}, \omega_{n}\right)$ are obtained from the Fourier transformation of $\Sigma_{\mathrm{S}}\left(\hat{i}, \omega_{n}\right)$ and $G\left(\hat{i}, \omega_{n}\right)$, respectively.

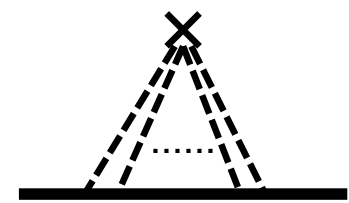

Fig. 2. Self-energy arising from the impurity scattering in the SCTMA

The self-energy arising from the disorder is given as,

$$
\Sigma_{\mathrm{d}}\left(\omega_{n}\right)=-\frac{n_{\mathrm{imp}}}{\sum_{k} G\left(\boldsymbol{k}, \omega_{n}\right)}
$$

where $n_{\text {imp }}=\left(N-N_{0}\right) / N$ is the impurity concentration. The diagrammatic representation of eq. (17) is shown in Fig. 2. Here, we have taken the limit $W \rightarrow \infty$ for simplicity, which is consistent with the sufficiently strong potential $W=40 t$ in $\S 2.1$. We have ignored the vertex correction arising from the disorder on the T-matrix because it is negligible for the $d$-wave SC fluctuation. ${ }^{28,29}$

If we neglect the self-energy due to the SC fluctuation $\Sigma_{\mathrm{S}}\left(\boldsymbol{k}, \omega_{n}\right)$, this calculation is reduced to the SCTMA in the mean field theory. The deviation from the pure $d$ wave superconductivity in the experimental results, such as the NMR $1 / T_{1} T$ and magnetic field penetration depth, has been resolved within the mean field theory. ${ }^{50-52}$ The consistency between the theory and experiment has been regarded as an evidence for the $d$-wave pairing in high- $T_{\mathrm{c}}$ cuprates. ${ }^{53,54}$ We show that this approximation breaks down in the under-doped region, while the SCTMA will be valid in the over-doped and electron-doped region.

\section{Microscopic Inhomogeneity and SC Fluctua- tion}

\subsection{Microscopic inhomogeneity}

First of all, we show the typical spatial dependence of SC order parameter in the disordered short coherence length superconductor. In order to discuss the long range correlation, we show the results of non-self-consistent $\mathrm{T}$ matrix approximation for $N=65 \times 65$ sites in this subsection. We have confirmed that qualitatively the same results are obtained in the self-consistent calculation.

We determine the spatial dependence of SC order parameter from the fluctuation propagator, namely the $\mathrm{T}$ matrix. Although the amplitude of SC order parameter is not determined by the T-matrix, the spatial dependence is determined in the following way. First, we solve the eigenvalue equation to diagonalize the irreducible $\mathrm{T}$ matrix as,

$$
t_{n} v(n, \hat{i}, \hat{\delta})=T_{0}\left(\hat{i}, \hat{j}, \hat{\delta}, \hat{\delta}^{\prime}\right) v\left(n, \hat{j}, \hat{\delta}^{\prime}\right) .
$$

Here, $t_{n}$ is the eigenvalue of $T_{0}\left(\hat{i}, \hat{j}, \hat{\delta}, \hat{\delta}^{\prime}\right)$ and we define $t_{1} \geq t_{2} \geq t_{3} \ldots \ldots \geq t_{4 N}$. Then, the T-matrix is described as,

$$
T\left(\hat{i}, \hat{j}, \hat{\delta}, \hat{\delta}^{\prime}\right)=\sum_{n} v(n, \hat{i}, \hat{\delta}) \frac{t_{n}}{1+V t_{n}} v\left(n, \hat{j}, \hat{\delta}^{\prime}\right),
$$

and the critical temperature is determined by the criterion $|V| t_{1}=1$. The order parameter of $d$-wave superconductivity just below $T_{\mathrm{c}}$ is obtained as,

$$
\Delta_{\mathrm{d}}(\hat{i})=\sum_{\delta}(-1)^{P} v(1, \hat{i}, \hat{\delta}),
$$

where $P=0(P=1)$ for $\hat{\delta}=( \pm 1,0)(\hat{\delta}=(0, \pm 1))$. The extended $s$-wave component of the order parameter is also determined as,

$$
\Delta_{\mathrm{s}}(\hat{i})=\sum_{\delta} v(1, \hat{i}, \hat{\delta})
$$

Although $\Delta_{\mathrm{S}}(\hat{i})$ is finite in the disordered system, it is much smaller than the $d$-wave component $\Delta_{\mathrm{d}}(\hat{i})$. The small $s$-wave component does not play any important role in the following results.

We show the typical spatial dependence of $\Delta_{\mathrm{d}}(\hat{i})$ in Fig. 3. We choose the parameter as $V=-0.6$ in Fig. 3(a) while we choose $V=-1.5$ in Figs. $3(\mathrm{~b})$ and (c). The disorder concentration is chosen to be $n_{\text {imp }}=0.01$ in Figs. 3(a) and (b) and $n_{\text {imp }}=0.05$ in Fig. 3(c). Note that the coherence length in the clean limit $\xi_{0}$ is scaled by $T_{\mathrm{c}}^{0}$ as $\xi_{0} \sim v_{\mathrm{F}} / T_{\mathrm{c}}^{0}$ where $T_{\mathrm{c}}^{0}$ is the transition temperature in the clean limit and $v_{\mathrm{F}}$ is the Fermi velocity. We obtain $T_{\mathrm{c}}^{0}=0.0418$ and $T_{\mathrm{c}}^{0}=0.269$ for $V=-0.6$ and $V=-1.5$, respectively. Thus, the coherence length in Fig. 3(a) is much longer than that in Figs. 3(b) and (c).

We clearly see the remarkable spatial inhomogeneity of SC order parameter in Figs. 3(b) and (c). In particular, the SC order parameter is highly localized by the $5 \%$ disorder-doping in the case of $V=-1.5$. It should be noticed that these results are very unusual. In case of the conventional long coherence length superconductor, the $\mathrm{SC}$ order parameter is almost uniform in the microscopic scale as shown in Fig. 3(a). If we increase the disorder with fixing $V=0.6$, the superconductivity is depressed before the SC order parameter is localized. This is the usual behavior of non- $s$-wave superconductor where the superconductivity is suppressed by the non-magnetic impurities. Then, the inhomogeneity is expected only in the larger scale.

We understand these results by considering the two typical scales of impurity concentration. One is the critical concentration defined by the Abrikosov-Gorkov theory. ${ }^{49-52}$ The other is defined so that the mean length between impurities becomes comparable with the coherence length in the clean limit, namely $\xi_{0}$. The former is scaled as $n_{\text {imp }} \propto T_{\mathrm{c}}^{0} / E_{\mathrm{F}}$ where $E_{\mathrm{F}}$ is the Fermi energy. The latter is obtained as $n_{\mathrm{imp}} \sim \xi_{0}^{-2} \propto\left(T_{\mathrm{c}}^{0} / E_{\mathrm{F}}\right)^{2}$ in two 

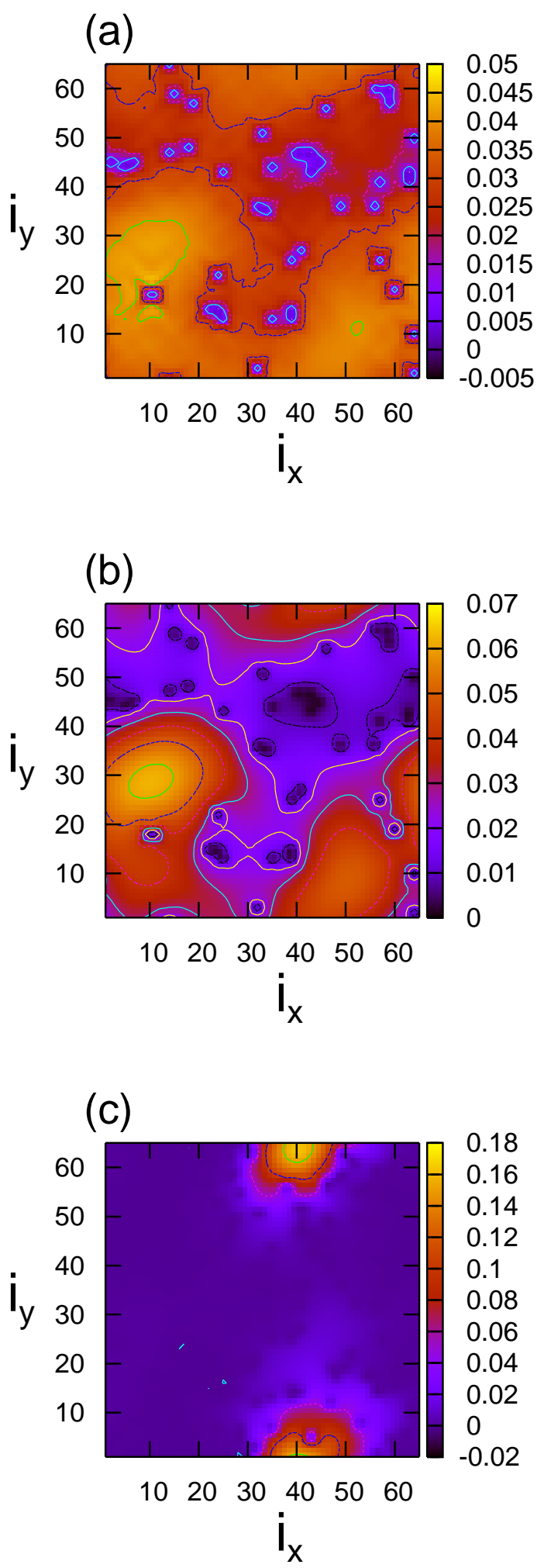

Fig. 3. Spatial dependence of $d$-wave SC order parameter at $T=$ $T_{\mathrm{c}}^{\mathrm{MF}}$. (a) The case of long coherence length superconductor, $V=$ -0.6 and $n_{\text {imp }}=0.01$. (b) The case of short coherence length superconductor, $V=-1.5$ and $n_{\mathrm{imp}}=0.01$. (c) $V=-1.5$ and $n_{\text {imp }}=0.05$. dimension. The latter is much smaller than the former in the low temperature superconductors, and therefore the system has many impurities in the area $\sim \xi_{0}^{2}$ at the moderate impurity concentration. This is the underlying reason why the Abrikosov-Gorkov theory is justified.

In contrast to the conventional case, the two scales can be comparable in the short coherence length superconductor, such as the high- $T_{\mathrm{c}}$ and organic superconductors. Then, the local superconductivity is stabilized in the "clean region" where only a few impurities exist. For example, we show the distribution of point disorders for $n_{\text {imp }}=0.05$ in Fig. 4(a). Comparing with Fig. 3(c), we see that the SC order parameter is enhanced in the nanoscale "clean region" which is shown by the red circles. The SC order parameter in Fig. 3(c) is localized in one of the red circles in Fig. 4(a). We understand that the SC correlation develops in the other "clean regions" by showing $\Delta_{2}(\hat{i})=\sum_{\delta}(-1)^{P} v(2, \hat{i}, \hat{\delta})$ and $\Delta_{3}(\hat{i})=\sum_{\delta}(-1)^{P} v(3, \hat{i}, \hat{\delta})$ which are the $d$-wave order parameters having the second and third highest $T_{\mathrm{c}}$, respectively. It is shown in Figs. 4(b) and (c) that they are developed in one of the red circles in Fig. 4(a). Thus, the highly disordered $d$-wave superconductor with short coherence length has a granular-like spatial structure. It should be stressed that the length scale of each granular is nanometer scale which is much smaller than the conventional granular systems with macroscopic or mesoscopic scale.

The microscopic inhomogeneity in Figs. 3(b), 3(c) and 4 is consistent with the STM measurement in the underdoped region. ${ }^{19}$ This experimental observation is the fingerprint of the $d$-wave short coherence length superconductor with point-like disorders. This interpretation is consistent with the recent experimental report ${ }^{20}$ where the spatial structure of SC order parameter is associated with the extended but point impurities. Then, the clean region is characterized by the small gap and the sharp gap edge. ${ }^{55}$ The broad gap structure in the dirty region should be attributed to the competing order. ${ }^{56,57}$

As a result of the nanoscale inhomogeneity of SC order parameter, the mean field value of transition temperature $T_{\mathrm{c}}^{\mathrm{MF}}$ is robust for the disorder, as pointed out in Ref. 58 . This is simply because the localized order parameter in the "clean region" is little affected by the disorder potential. We numerically take the random average of transition temperature $\bar{T}_{\mathrm{c}}^{\mathrm{MF}}$ and show the result in Fig. 5. The result of SCTMA is also shown for a comparison. The decrease of the mean field transition temperature remarkably deviates from the SCTMA in case of the large $|V|$, namely the short coherence length $(V=-1.5)$ while the deviation is negligible in case of the long coherence length $(V=-0.6)$. Thus, the Abrikosov-Gorkov theory breaks down in the presence of the nanoscale inhomogeneity.

Before closing this subsection, we note that the spatial dependence of $\mathrm{SC}$ order parameter is quite different in the $s$-wave superconductor. Then, the order parameter is almost uniform for $10 \%$ disorder-doping even if the coherence length is short. ${ }^{59}$ This is because the $s$-wave superconductivity is robust for the non-magnetic impurity as proved by Anderson. ${ }^{60}$ We obtain the spatial depen- 
(a)

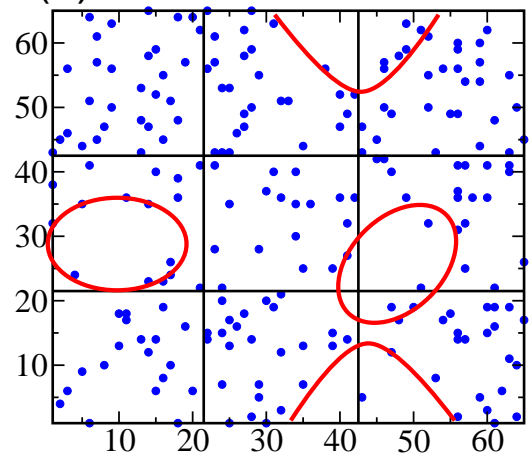

(b)

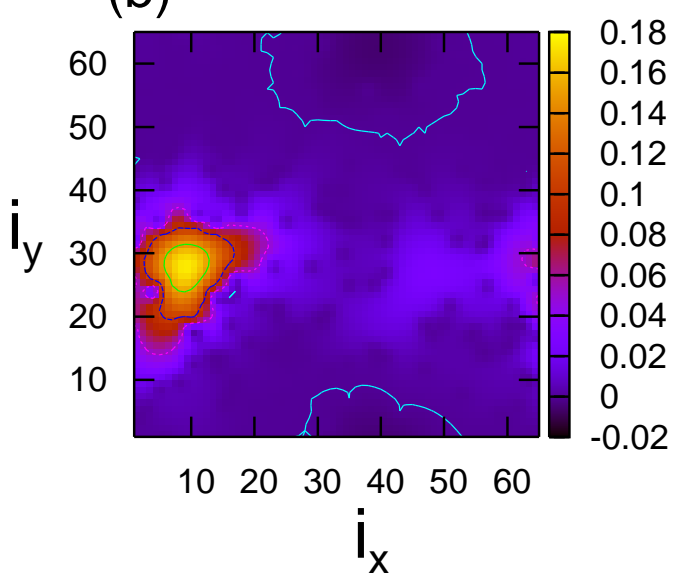

(c)

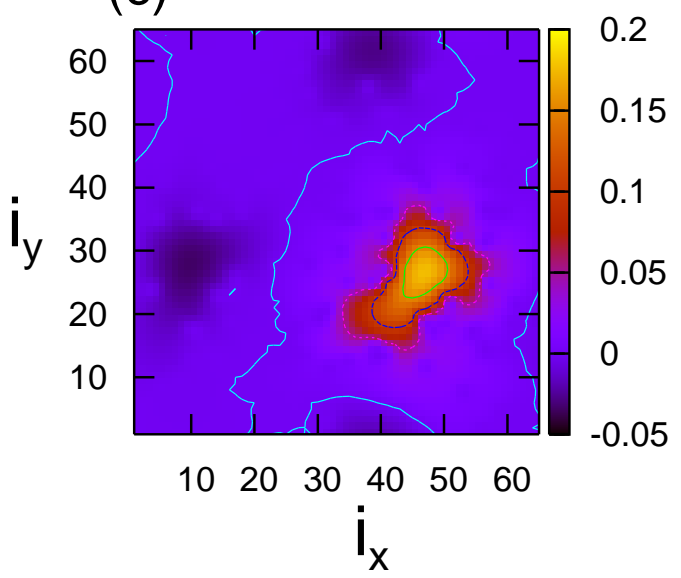

Fig. 4. (a) The distribution of point disorders in Fig. 3(c). We show the "clean region" by red circles. The SC correlation is locally enhanced in these regions. (b) Spatial dependence of $\Delta_{2}(\hat{i})$ and (c) that of $\Delta_{3}(\hat{i})$. We choose the same parameters as in Fig. 3(c).

dence similar to Fig. 3(a) where the SC order parameter is nearly homogeneous except for the abrupt suppression around impurities. Thus, the $d$-wave pairing symmetry plays an essential role in the inhomogeneous structure of $\mathrm{SC}$ order parameter in the nanometer scale. This may be the reason why the critical behavior in short coherence length $s$-wave superconductor $\mathrm{NbN}$ is quite different

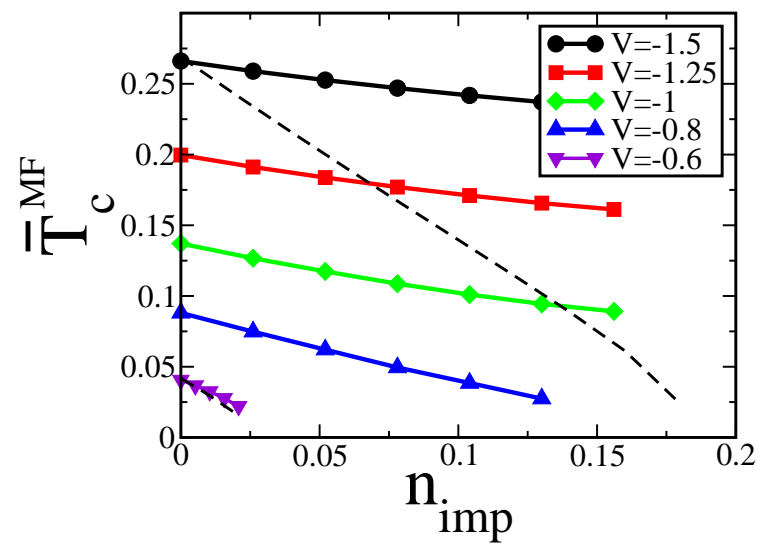

Fig. 5. Random average of the transition temperature $\bar{T}_{\mathrm{c}}^{\mathrm{MF}}$ in the mean field theory. Here, we take more than 50 samples for the random average. The attractive interaction is varied as $V=$ $-0.6,-0.8,-1,-1.25$ and -1.5 from the bottom to the top. We show the results of SCTMA with $V=-0.6$ and $V=-1.5$ (dashed lines) for a comparison.

from that in the under-doped cuprates. ${ }^{61}$

\subsection{SC fluctuation in the inhomogeneous system}

The purpose of this paper is to investigate the SC fluctuation in the disordered system with focus on the role of microscopic inhomogeneity. We discuss this issue by showing the results of self-consistent T-matrix approximation.

First, we discuss the transition temperature of superconductivity. Because the long range order is not realized at finite temperature in purely two-dimensional systems according to the Mermin-Wagner theorem, we adopt a phenomenological procedure to include the weak three dimensionality. For this aim, the criterion $|V| t_{1}=1-\delta$ has been adopted instead of $|V| t_{1}=1$ when the clean system has been investigated. ${ }^{14}$ We also adopt this procedure and determine the $T_{\mathrm{c}}$ for $n_{\mathrm{imp}}=0$ by choosing $\delta=0.02$. However, it is not clear whether this criterion is applicable to the disordered systems. In particular, this procedure may break down in the disordered short coherence length superconductor with nanoscale inhomogeneity. Then, it is expected that the long range correlation hardly develops although the local superconductivity occurs as in Figs. 3 and 4. Therefore, we determine the $T_{\mathrm{c}}$ by the following procedure. First, we calculate the correlation function of $d$-wave SC order parameter $\bar{\chi}_{\mathrm{d}}(\hat{r})$, which is defined as,

$$
\begin{gathered}
\chi_{\mathrm{d}}(\hat{i}, \hat{j})=\sum_{\delta, \delta^{\prime}}(-1)^{P+P^{\prime}} T\left(\hat{i}, \hat{j}, \hat{\delta}, \hat{\delta}^{\prime}\right), \\
\bar{\chi}_{\mathrm{d}}(\hat{r})=<\frac{1}{N} \sum_{i} \chi_{\mathrm{d}}(\hat{i}+\hat{r}, \hat{i})>_{\mathrm{r}},
\end{gathered}
$$

where $<>_{\mathrm{r}}$ denotes the random average. Here, $P\left(P^{\prime}\right)$ is determined by $\hat{\delta}\left(\hat{\delta}^{\prime}\right)$ as in eq. (20). We denote the correlation function for $n_{\text {imp }}=0$ and $|V| t_{1}=0.98$ as $\chi_{\mathrm{d}}^{(\mathrm{c})}(\hat{r})$ and adopt the criterion,

$$
\bar{\chi}_{\mathrm{d}}(15,15)=\chi_{\mathrm{d}}^{(\mathrm{c})}(15,15),
$$


to determine the $T_{\mathrm{c}}$ in disordered systems. Note that $\hat{r}=(15,15)$ is the longest length in the calculation for $N=31 \times 31$ with (anti-)periodical boundary condition. This criterion determines the temperature where the long range correlation begins to develop.
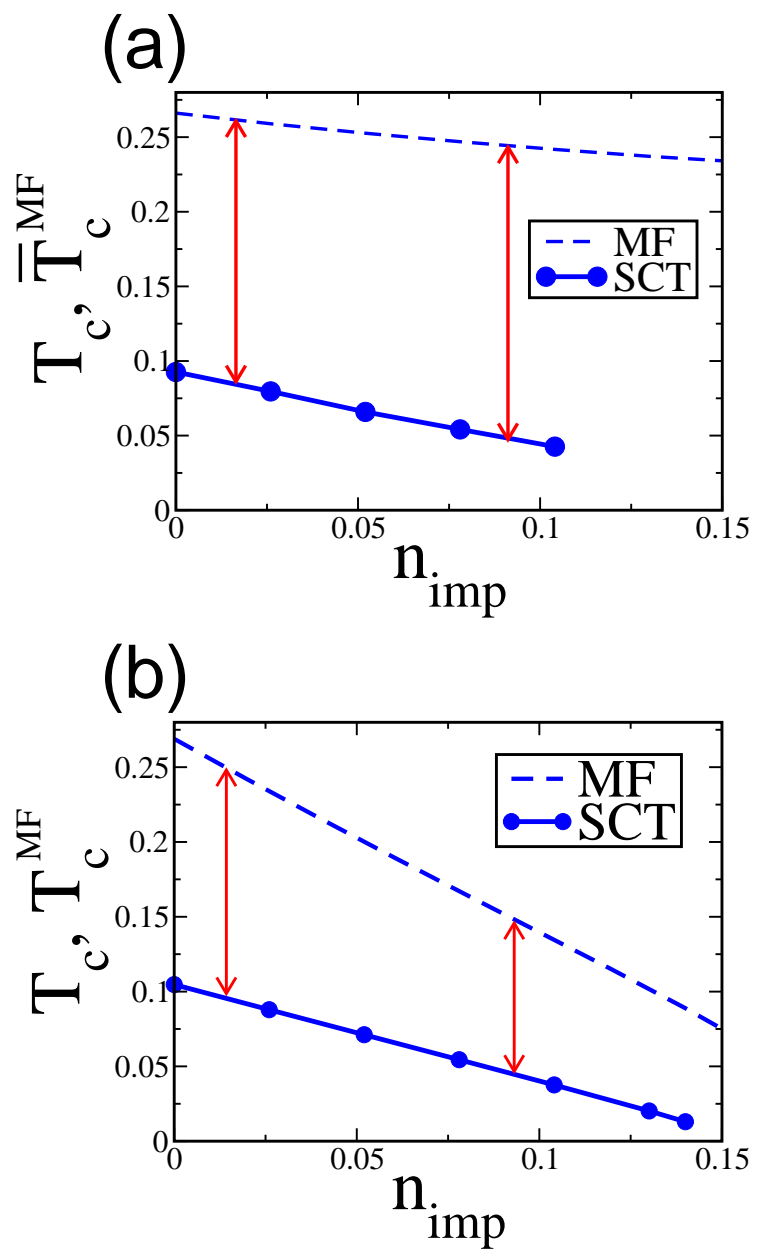

Fig. 6. The transition temperature obtained in the self-consistent T-matrix approximation for $V=-1.5$. (a) The results of the formulation in $\S 2.1$ where the disorder is exactly taken into account. We take at least 20 samples and take the random average to estimate the correlation function, eq. (23). (b) The results of SCTMA. We show the transition temperature in the mean field theory, $\bar{T}_{\mathrm{c}}^{\mathrm{MF}}$ and $T_{\mathrm{c}}^{\mathrm{MF}}$ by the dashed lines.

We show the results of $T_{\mathrm{c}}$ in Fig. 6(a). The results obtained in the SCTMA are shown in Fig. 6(b) for a comparison. The same criterion, namely eq. (24) is adopted in the SCTMA. The transition temperatures in the mean field theory ((a) $\bar{T}_{\mathrm{c}}^{\mathrm{MF}}$ and (b) $T_{\mathrm{c}}^{\mathrm{MF}}$, respectively) are shown to illuminate the role of SC fluctuation.

Since the short range SC fluctuation develops below the mean field transition temperature, $\bar{T}_{\mathrm{c}}^{\mathrm{MF}}$ can be regarded as $T^{*}$ which is the onset of pseudogap phenomena arising from the SC fluctuation. An important value is the difference $\bar{T}_{\mathrm{c}}^{\mathrm{MF}}-T_{\mathrm{c}}$ which measures the width of critical region. When the SC fluctuation is strong, the critical region is large and vice versa.

We clearly see the qualitatively different behaviors of critical region between Figs. 6(a) and (b). The results of the formulation in $\S 2.1$ show that the critical region is $e n$ larged by the disorder. However, the SCTMA provides an opposite result. This discrepancy means that the result of Fig. 6(b) is an artifact of the SCTMA because the disorder is exactly taken into account in Fig. 6(a). This comparison illuminates the breakdown of Abrikosov-Gorkov theory for the fluctuation phenomena in the short coherence superconductor. ${ }^{62}$

The result of SCTMA, where the SC fluctuation is suppressed by the disorder, is seemingly reasonable because the $T_{\mathrm{c}}$ is decreased by the disorder. However, the microscopic inhomogeneity neglected in the SCTMA enhances the fluctuation and disturbs the long range order. The latter effect is significant in the short coherence length superconductor like under-doped cuprates and organic superconductor $\kappa$-(BEDT-TTF $)_{2} \mathrm{X}$. Thus, the enhancement of SC fluctuation indicated by the experimental results $^{30-32}$ is explained by appropriately taking into account the inhomogeneous structure of SC fluctuation.
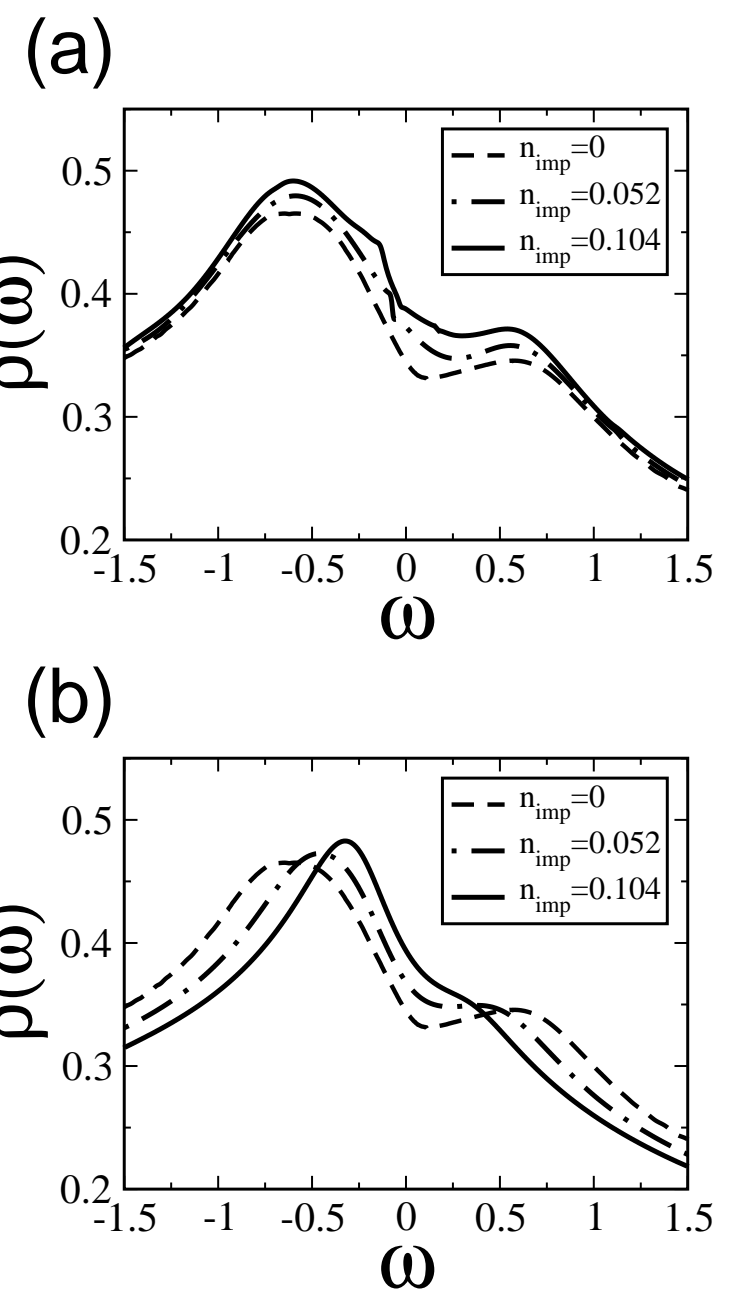

Fig. 7. The spatially averaged $\operatorname{DOS} \rho(\omega)$ for $V=-1.5$. (a) The results of the formulation in $\S 2.1$. We take more than 20 samples to take the random average. (b) The results of SCTMA.

The experiments ${ }^{30-32}$ have shown that the pseudogap is robust for the disorder even though the transition temperature is significantly decreased. In order to illuminate 
this feature, we show the single particle DOS at $T=T_{\mathrm{c}}$. We calculate the local DOS $\rho(\hat{r}, \omega)$ in each sample which is defined as,

$$
\rho(\hat{r}, \omega)=-\frac{1}{\pi} \operatorname{Im} G^{\mathrm{R}}(\hat{r}, \hat{r}, \omega),
$$

where the retarded Green function is obtained by the Pade approximation. By taking the spatial average and random average, we obtain the DOS per $\mathrm{Cu}$ sites as,

$$
\rho(\omega)=<\frac{1}{N_{0}} \sum_{r} \rho(\hat{r}, \omega)>_{\mathrm{r}} .
$$

As shown in Fig. 7(a), the pseudogap in the DOS is not significantly destroyed even though the $T_{\mathrm{c}}$ is remarkably reduced. This result should be contrasted to that of SCTMA where the pseudogap is smeared by the same amount of disorder (Fig. 7(b)). Thus, the robust pseudogap indicated by the experiments is attributed to the role of microscopic inhomogeneity.

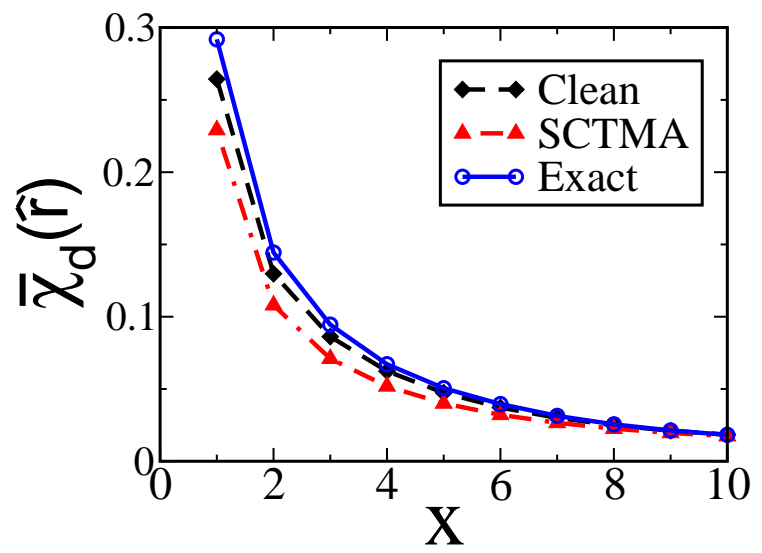

Fig. 8. The correlation function of SC order parameter $\bar{\chi}_{\mathrm{d}}(\hat{r})$. We show the spatial dependence along the diagonal line $\hat{r}=$ $(x, x)$. The solid line is the result of the calculation formulated in $\S 2.1$ and the dash-dotted line shows the result of SCTMA for $n_{\text {imp }}=0.104$. The dashed line is the result in the clean limit. We choose $V=-1.5$ and the temperature which satisfies the criterion eq. (24). Note that the correlation functions at large $x$ are similar to each other owing to the criterion.

In order to understand the results in Figs. 6 and 7 more clearly, we again stress that the short range SC correlation develops even though the long range coherence is disturbed by the localization of order parameter. Because we have defined the $T_{\mathrm{c}}$ as the temperature where the long range correlation begins to develop, the short range correlation at that temperature is enhanced by the disorder. This feature is clearly shown in Fig. 8 which shows the correlation function of SC order parameter, namely $\bar{\chi}_{\mathrm{d}}(\hat{r})$. The pseudogap in the short coherence length superconductor is robust for the disorder since the pseudogap is mostly induced by the short range correlation. ${ }^{14}$ We note that the qualitatively different results are obtained in the SCTMA where the short range correlation is reduced by the disorder. This is because the coherence length increases owing to the decrease of $T_{\mathrm{c}}$ and the long range correlation tends to develop. This feature is consistent with Fig. 7(b), but an artifact of the SCTMA.

It should be noted that the long range correlation is still weak in Fig. 8. This means that the actual long range coherence is achieved at the temperature lower than $T_{\mathrm{c}}$ in our definition. In order to discuss the true long range order, we have to take the weak three dimensionality into account. The nature of dimensional crossover from $2 \mathrm{D}$ to $3 \mathrm{D}$ has been investigated in the clean systems. Then, the qualitative behaviors of $T_{\mathrm{c}}$ do not depend on the phenomenological criterion. ${ }^{46}$ However, the nature of long range order is not clear in the highly disordered system. It is expected from Figs. 3 and 4 that the long range order is triggered by the phase coherence between each "clean region" like granular superconductivity. ${ }^{63}$ The result in Fig. 8 is consistent with this picture although the calculation with $N=31 \times 31$ is not sufficient to describe the phase correlation between each granular. We have confirmed that the behaviors in Figs. 6 and 8 become notable as increasing the size of calculation. It is an interesting future problem to develop a theory on the crossover from the homogeneous superconductivity to the granular superconductivity.

\section{Pseudogap around Single Impurity}

In $\S 3$, we have investigated the role of microscopic inhomogeneity on the macroscopic properties of $d$-wave short coherence length superconductor. We briefly discuss the spatial dependence in the atomic scale in this section.

Here, we focus on the spatial dependence of the electronic DOS in the presence of strong point disorders like Zn impurities. Because the most significant spatial dependence is the variation around the disorder, we show the DOS around the single impurity. The pseudogap around the single impurity has been investigated by the NMR measurements. ${ }^{64-67}$ We discuss the relevance of our results for these experiments.

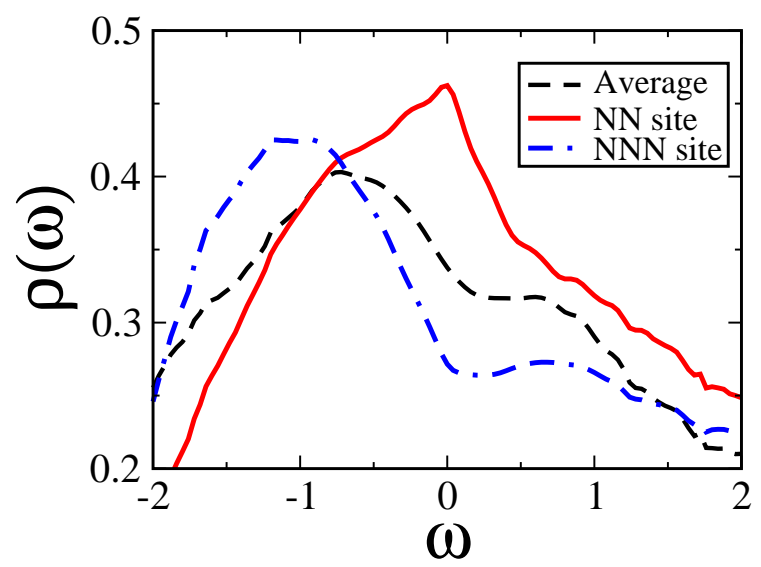

Fig. 9. The LDOS around the single impurity. We show the LDOS at the nearest neighbor (NN) site (solid line) and next nearest neighbor $(\mathrm{NNN})$ site (dot-dashed line) in addition to the spatially averaged DOS (dashed line).

In this section, we adopt the self-consistent T-matrix approximation for $N=31 \times 31$ sites. Figure 9 shows the 
local DOS (LDOS) at the nearest neighbor (NN) and next nearest neighbor (NNN) sites where the deviation from the averaged DOS is most significant. The temperature is chosen to be slightly above $T_{\mathrm{c}}$ and therefore the pseudogap opens in the averaged DOS as shown by the dashed line. It is clearly shown that the pseudogap is destroyed at the NN site. This is because the Cooper pairing is forbidden between the impurity site and NN site. We have confirmed that the $d$-wave order parameter is remarkably suppressed at the NN site. The destroyed pseudogap at the NN site is consistent with the NMR measurements which have shown the Curie-Weiss enhancement of $1 / T_{1} T$ around the non-magnetic impurity. ${ }^{64-67}$ The neutron scattering measurements have also shown the destruction of superconducting gap in the magnetic excitation. ${ }^{68,69}$

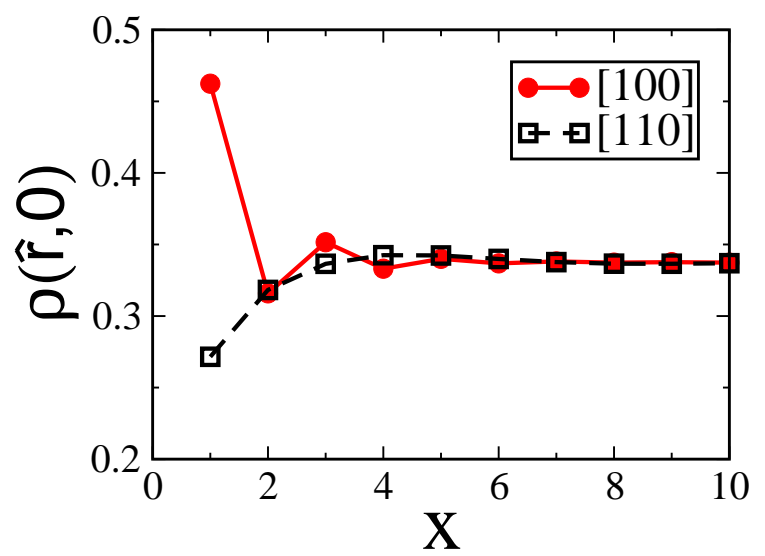

Fig. 10. The spatial dependence of the LDOS along the [100] and [110] directions from the impurity site. We define the impurity site $\hat{r}=(0,0)$ and show the LDOS $\rho(\hat{r}, 0)$ at $\hat{r}=(x, 0)$ (circles) and $\hat{r}=(x, x)$ (squares), respectively.

Figure 9 shows that the pseudogap in the DOS at the NNN site is larger than that in the averaged DOS. Thus, the spatial dependence of the DOS is non-monotonic. We show the LDOS $\rho(\hat{r}, 0)$ at the Fermi energy along [100] and [110] directions in Fig. 10. The DOS remarkably oscillates along [100] direction while the DOS shows a long wave length oscillation along [110] direction. It should be noted that the SC order parameter is monotonically suppressed around the impurity site and shows no oscillation. Thus, the suppression of the DOS along the [110] direction is not attributed to the enhancement of the SC correlation. Actually, the oscillation of the DOS is a kind of the quasi-particle interference effect ${ }^{70}$ which reflects the shape of the Fermi surface.

We have analyzed the magnetic excitations in the disordered $t-t^{\prime}-U-V$ model in order to take into account the competing anti-ferromagnetic fluctuation ${ }^{71}$ in addition to the SC fluctuation. Then, we found that the spatial dependences of static quantities are quite different from that of NMR $1 / T_{1} T$. The staggard spin susceptibility is monotonically enhanced near the impurity. This enhancement of the anti-ferromagnetic correlation induces the rapid oscillation of uniform spin susceptibil- ity which has been observed in the Knight shift measurements. ${ }^{72}$ This spatial dependence of the static antiferromagnetic correlation can be qualitatively described by the Ginzburg-Landau theory which assumes the competition between the anti-ferromagnetism and superconductivity. In contrast to that, the $1 / T_{1} T$ shows a spatial dependence similar to the electronic DOS in Fig. 10, which can not be described in the Ginzburg-Landau theory. This is consistent with the experimental result on the $1 / T_{1} T$ which shows the length scale shorter than the Ginzburg-Landau correlation length. ${ }^{73}$ The details will be shown in the future publication. ${ }^{56}$

When we distribute many strong point disorders like Figs. 3 and 4, the Fourier transformed DOS (FTDOS) is dominated by the deviation at the NN and NNN sites. Then, no clear structure appears in the FTDOS around $q=(\pi / 4,0)$. In contrast to that, the peak appears in the FTDOS around $q=(\pi / 4,0)$ (not shown) for the weak random disorder. The latter is similar to the experimental observation in the pseudogap state, ${ }^{74}$ although the structure around $q=(\pi / 4,0)$ is smeared in the high frequency region.

We note that the nanoscale inhomogeneity of DOS observed in the $\mathrm{STM}^{19-21}$ is not clearly reproduced in this calculation. We consider that the huge and broad gap structure in the weakly superconducting region is due to the correlation competing with the $d$-wave superconductivity. ${ }^{57} \mathrm{~A}$ candidate is the disordered magnetism observed in the neutron, $\mu \mathrm{SR}$ and NMR measurements. ${ }^{22-26}$

\section{Discussion}

\subsection{Summary and discussion}

We have investigated the disorder-induced microscopic inhomogeneity of SC order parameter and its effects on the pseudogap in short coherence length superconductors with $d$-wave symmetry. We find that the SC order parameter is highly localized by a small amount of point disorders and the nanoscale inhomogeneity like a granular superconductor appears. Owing to the spatially localized SC correlation, the long range coherence hardly develops while the short range correlation is enhanced. As a result, the critical region of superconductivity is enlarged by the disorder in contrast to the SCTMA based on the Abrikosov-Gorkov theory. ${ }^{28,29}$ Then, the pseudogap induced by the SC fluctuation is robust for the disorders.

One of the typical short coherence length superconductors is the high- $T_{\mathrm{c}}$ superconductor. The under-doped cuprates satisfy three conditions to realize the microscopic inhomogeneity, namely the short coherence length, non-s-wave symmetry and quasi-two dimensionality. Actually, the nanoscale inhomogeneity has been observed in the STM measurements ${ }^{19-21}$ and also in the Josephson plasma resonance measurement. ${ }^{75}$ We note that the disorder induces the inhomogeneity of SC order parameter without remarkable charge inhomogeneity. The results of NMR and transport measurements ${ }^{30-32,62}$ indicating the breakdown of Abrikosov-Gorkov theory are consistent with the microscopic inhomogeneity induced by the disorder. We understand these experimental results in a 
coherent way by appropriately taking the spatial dependence into account.

It should be stressed that there are intrinsic disorders in high- $T_{\mathrm{c}}$ cuprates. Therefore, our results indicate the nanoscale inhomogeneity in the non-disorder-doped sample. Then, it is expected that the superconducting transition in the highly under-doped region is triggered by the phase coherence of nanoscale granular regions. This picture is consistent with the Uemura $\operatorname{plot}^{76}$ which has implied the importance of phase fluctuation as pointed out by Emery and Kievelson. ${ }^{6}$ The recent experiments have also indicated the two dimensional phase fluctuation above $T_{\mathrm{c}} \cdot{ }^{61,77,78}$

The nature of the SI transition in the highly underdoped region is beyond the scope of this paper. However, it is reasonable to consider that the disordered magnetism occurs as shown by the experiments. ${ }^{22-25}$ We will discuss this topic in another publication. ${ }^{56}$

\subsection{Discussion on a variety of SI transitions}

A variety of SI transitions have been observed in strongly correlated electron systems. Here, we briefly discuss how the differences can be understood by considering the role of disorder. The importance of disorder in the under-doped cuprates is illuminated by the following discussion.

The nature of SI transition is different between the series of high- $T_{\mathrm{c}}$ cuprates. The disordered magnetism has been observed most clearly in the La-based systems where the $\mathrm{CuO}_{2}$ layer is significantly affected by the disorder. In contrast to that, the recent work on the multilayer cuprates has shown the abrupt change from the superconducting state to the anti-ferromagnetic metallic state at finite temperatures. ${ }^{79}$ The latter is consistent with the theoretical results based on the FLEX and FLEX+T-matrix approximations in the clean system. ${ }^{14}$ The authors have claimed that the clean $\mathrm{CuO}_{2}$ layer is realized in this compound. ${ }^{79}$ Then, the absence of the disordered magnetism and/or the anti-ferromagnetic insulating state implies that the disorder plays an essential role for the SI transitions in the other cuprates.

The organic superconductor $\kappa$-(BEDT-TTF $)_{2} \mathrm{X}^{80}$ is another candidate for the short coherence length $d$-wave superconductor. ${ }^{14,81}$ It is expected that the system is relatively clean because the material is free from the dopant disorders. The first order phase transition from the superconductivity to the anti-ferromagnetism indicates that the organic superconductor $\kappa$-(BEDT-TTF $)_{2} \mathrm{X}$ is analogous with the multi-layer high- $T_{\mathrm{c}}$ cuprates.

The electron-hole asymmetry of the SI transition in cuprate superconductors is understood by considering the difference of coherence length. The result of the FLEX+T-matrix approximation has shown that the coherence length is much longer in the electron-doped region than in the hole-doped region. ${ }^{34}$ This is the reason why the pseudogap due to the SC fluctuation is not observed in the electron-doped cuprates. ${ }^{82-84}$ Then, the abrupt change from the superconducting state to the anti-ferromagnetic state ${ }^{85}$ is attributed to the absence of microscopic inhomogeneity which is unlikely in the long coherence length superconductor.
A variety of SI transitions accompanying the magnetism have been discovered in the heavy fermion materials. ${ }^{86}$ It is reasonable to consider that the multi-orbital effect plays an essential role for the phase transition from the superconductivity to the magnetism. In some cases, the superconductivity and magnetism are induced by the different orbitals. Then, the coupling between the superconductivity and magnetism is weak and the co-existent state can be stabilized.

\subsection{Plan of the future study}

It is a challenging future work to describe the SI transition on the basis of the disorder-induced nanoscale inhomogeneity. ${ }^{87}$ Some theoretical developments are needed for this aim.

First, it is important to take into account the competition between the superconductivity and antiferromagnetism. This competition induces the spatially inhomogeneous structure of superconducting region and magnetic region. ${ }^{88}$ We have examined the spatial structure on the basis of the $t-t^{\prime}-U-V$ model which includes the on-site repulsion. Then, we found that the magnetic correlation is enhanced in the "dirty region" where the superconducting correlation is destroyed by the disorder. The results will be shown in the future publication. ${ }^{56}$

Second, it is necessary to take into account the quantum fluctuation to describe the SI transition at zero temperature although we have investigated the thermal fluctuation in this paper. One of the promising strategies is to derive the phenomenological models such as the Ginzburg-Landau model or the XY model where the long range behaviors are illuminated. ${ }^{89}$ We are planning to microscopically derive a phenomenological model which is relevant in the spatially coexistent state of the superconductivity and anti-ferromagnetism.

\section{Acknowledgments}

The authors are grateful to T. Adachi, A. Fujimori, K. Fujida, M. Fujita, M. Ido, K. Ishida, Y. Itoh, H. Kitano, Y. Koike, H. Kontani, K. Kudo, N. Momono, H. Mukuda, M. Ogata, T. Shibauchi, K. Yamada and T. Yoshida for fruitful discussions. Numerical computation in this work was partly carried out at the Yukawa Institute Computer Facility. The present work has been partly supported by a Grant-In-Aid for Scientific Research from the Ministry of Education, Culture, Sport, Science and Technology of Japan.

1) For a review of experimental studies, T. Timusk and B. Statt: Rep. Prog. Phys. 62 (1999) 61.

2) B. Jankó, J. Maly and K. Levin: Phys. Rev. B 56 (1997) 11407; J. Maly, B. Jankó and K. Levin: Physica C 321 (1999) 113.

3) Y. Yanase and K. Yamada: J. Phys. Soc. Jpn. 68 (1999) 2999.

4) A. Kobayashi, A. Tsuruta, T. Matsuura and Y. Kuroda, J. Phys. Soc. Jpn. 68 (1999) 2506; ibid 70 (2001) 1214.

5) D. Rohe and W. Metzner, Phys. Rev. B 63 (2001) 224509.

6) V. J. Emery and S. A. Kivelson: Phys. Rev. Lett. 74 (1995) 3253.

7) M. Franz and A. J. Millis: Phys. Rev. B 58 (1998) 14572.

8) H-J. Kwon and A. T. Dorsey: Phys. Rev. B 59 (1999) 6438.

9) A. Paramekanti, M. Randeria, T. V. Ramakrishnan and S. S. Mandal: Phys. Rev. B 62 (2000) 6786 
10) V. M. Loktev, R. M. Quick and S. G. Sharapov: Phys. Rep. 349 (2001) 1.

11) T. Eckl, D. J. Scalapino, E. Arrigoni, and W. Hanke: Phys. Rev. B 66 (2002) 140510.

12) M. Franz and Z. Tesanovic, Phys. Rev. Lett. 87 (2001) 257003: 88 (2002) 109902 (E). Z. Tesanovic, O. Vafek, and M. Franz: Phys. Rev. B 65 (2002) 180511.

13) I. F. Herbut: Phys. Rev. B 70 (2004) 184507.

14) Y. Yanase, T. Jujo, T. Nomura, H. Ikeda, T. Hotta and K. Yamada: Phys. Rep. 387 (2004) 1.

15) Y. Yanase and K. Yamada: J. Phys. Soc. Jpn. 69 (2000) 2209.

16) Y.-J. Kao, A. P. Iyengar, Q. Chen and K. Levin: Phys. Rev. B 64 (2001) 140505(R).

17) K. Gorny, O. M. Vyaselev, J. A. Martindale, V. A. Nandor, C. H. Pennington, P. C. Hammel, W. L. Hults, J. L. Smith, P. L. Kuhns, A. P. Reyes, and W. G. Moulton: Phys. Rev. Lett. 82 (1999) 177.

18) G.-q. Zheng, W. G. Clark, Y. Kitaoka, K. Asayama, Y. Kodama, P. Kuhns and W. G. Moulton: Phys. Rev. B 60 (1999) R9947.

19) K. M. Lang, V. Madhavan, J. E. Hoffman, E. W. Hudson, H. Eisaki, S. Uchida and J. C. Davis: Nature 415 (2002) 412; S. H. Pan, J. P. O'Neal, R. L. Badzey, C. Chamon, H. Ding, J. R. Engelbrecht, Z. Wang, H. Eisaki, S. Uchida, A. K. Gupta, K.-W. Ng, E. W. Hudson, K. M. Lang and J. C. Davis: Nature 413 (2001) 282.

20) K. McElroy, Jinho Lee, J. A. Slezak, D.-H. Lee, H. Eisaki, S. Uchida and J. C. Davis: Science 309 (2005) 1048.

21) A. Sugimoto, S. Kashiwaya, H. Eisaki, H. Kashiwaya, H. Tsuchiura, Y. Tanaka, K. Fujita and S. Uchida: Phys. Rev. B 74 (2006) 094503.

$22)$ B. Keimer, N. Belk, R. J. Birgeneau, A. Cassanho, C. Y. Chen, M. Greven, and M. A. Kastner, A. Aharony, Y. Endoh, R. W. Erwin and G. Shirane: Phys. Rev. B 46 (1992) 14034.

23) S. Wakimoto and G. Shirane, Y. Endoh, K. Hirota, S. Ueki, K. Yamada, R. J. Birgeneau, M. A. Kastner, Y. S. Lee, P. M. Gehring and S. H. Lee: Phys. Rev. B 60 (1999) R769; M. Matsuda, M. Fujita, K. Yamada, R. J. Birgeneau, M. A. Kastner, H. Hiraka, Y. Endoh, S. Wakimoto and G. Shirane: Phys. Rev. B 62 (2000) 9148.

24) Ch. Niedermayer, C. Bernhard, T. Blasius, A. Golnik, A. Moodenbaugh and J. I. Budnick: Phys. Rev. Lett. 80 (1998) 3843.

25) S. Sanna, G. Allodi, G. Concas, A. D. Hillier R. De Renzi: Phys. Rev. Lett. 93 (2004) 207001.

26) K. Ishida, H. Aya, Y. Tokunaga, H. Kotegawa, Y. Kitaoka, M. Fujita and K. Yamada: Phys. Rev. Lett. 92 (2004) 257001.

27) In order to avoid confusions, we denote the self-consistent tmatrix approximation for the disorder as SCTMA in the following part while the T-matrix approximation on the SC fluctuation is denoted in extenso.

28) Q. Chen and J. R. Schrieffer: Phys. Rev. B 66 (2002) 014512.

29) K. Kudo and K. Yamada: J. Phys. Soc. Jpn. 73 (2004) 2219.

30) Y. Itoh, T. Machi, N. Watanabe, S. Adachi and N. Koshizuka: J. Phys. Soc. Jpn. 70 (2001) 1881.

31) R. Rullier-Albenque, R. Tourbot, H. Alloul, P. Lejay, D. Colson and A. Forget: Phys. Rev. Lett. 96 (2006) 067002.

32) Z. A. Xu, J. Q. Shen, S. R. Zhao, Y. J. Zhang and C. K. Ong: Phys. Rev. B 72 (2005) 144527.

33) For a review of ARPES studies, A. Damascelli, Z. Hussain and Z.-X. Shen: Rev. Mod. Phys. 75 (2003) 473; J. C. Campuzano, M. R. Norman, and M. Randeria: cond-mat/0209476.

34) Y. Yanase and K. Yamada: J. Phys. Soc. Jpn. 70 (2001) 1659.

35) Y. Yanase: J. Phys. Soc. Jpn 71 (2002) 278.

36) K. Fujita, T. Noda, K. M. Kojima, H. Eisaki and S. Uchida: Phys. Rev. Lett. 95 (2005) 097006.

37) P. A. Lee, T. M. Rice, and P. W. Anderson: Phys. Rev. Lett. 31 (1973) 462.

38) M. V. Sadovskii: Sov. Phys. JETP 39 (1974) 845 [Zh. Eksp. Teor. Fiz. 66 (1974) 1720]; Sov. Phys. Solid State 16 (1975) 1362 [Fiz. Tverd. Tela 16 (1974) 2504]; Sov. Phys. JETP 50 (1979) 989 [Zh. Eksp. Teor. Fiz. 77 (1979) 2070].

39) O. Tchernyshyov: Phys. Rev. B 56 (1997) 3372.

40) J. Schmalian, D. Pines and B. Stojković: Phys. Rev. Lett. 80 (1998) 3839; Phys. Rev. B 60 (1999) 667.
41) O. Tchernyshyov: Phys. Rev. B 59 (1999) 1358.

42) L. Bartosch and P. Kopietz: Phys. Rev. B 60 (1999) 15488.

43) E. Z. Kuchinskii and M. V. Sadovskii: JETP 88 (1999) 968. [Zh. Eksp. Teor. Fiz. 115 (1999) 1765.]

44) A. J. Millis and H. Monien: Phys. Rev. B 61 (2000) 12496.

45) H. Monien: Phys. Rev. Lett. 87 (2001) 126402.

46) Y. Yanase: J. Phys. Soc. Jpn 73 (2004) 1000.

47) E. Dagotto: Rev. Mod. Phys. 66 (1994) 763.

48) N. Bulut: Adv. Phys. 51 (2002) 1587.

49) A. A. Abrikosov and L. P. Gor'kov: Sov. Phys. JETP 12 (1961) 1243.

50) T. Hotta: J. Phys. Soc. Jpn. 62 (1993) 274.

51) P. J. Hirschfeld and N. Goldenfeld: Phys. Rev. B 48 (1993) 4219.

52) Y. Sun and K. Maki: Phys. Rev. B 51 (1995) 6059.

53) K. Ishida, Y. Kitaoka, N. Ogata, T. Kamino, K. Asayama, J. R. Cooper and N. Athanassopoulou: J. Phys. Soc. Jpn. 62 (1993) 2803; Y. Kitaoka, K. Ishida, and K. Asayama: J. Phys. Soc. Jpn. 63 (1994) 2052.

54) W. N. Hardy, D. A. Bonn, D. C. Morgan, R. Liang and K. Zhang: Phys. Rev. Lett. 70 (1993) 3999.

55) This interpretation is also implied by the direct observation of the vortex trap. N. Fukuo, H. Mashima, Y. Matsumoto, T. Hitosugi and T. Hasegawa: Phys. Rev. B 73 (2006) 220505.

56) Y. Yanase and M. Ogata: To appear in J. Mag. Mag. Materials; Y. Yanase: in preparation.

57) A different intrepretation has been given in T. S. Nunner, B. M. Andersen, A. Melikyan and P. J. Hirschfeld: Phys. Rev. Lett. 95 (2005) 177003.

58) M. Franz, C. Kallin, A. J. Berlinsky, and M. I. Salkola: Phys. Rev. B 56 (1997) 7882; A. Ghosal, M. Randeria and N. Trivedi: Phys. Rev. B 63 (2000) 020505.

59) H. Yorozu, Y. Yanase and M. Ogata: unpublished.

60) P. W. Anderson: J. Phys. Chem. Solids 11 (1959) 26.

61) H. Kitano, T. Ohashi, A. Maeda and I. Tsukada: Phys. Rev. B 73 (2006) 092504; T. Ohashi, H. Kitano, A. Maeda, H. Akaike and A. Fujimaki: Phys. Rev. B 73 (2006) 174522.

62) For a recent experimantal evidence for the breakdown of AG theory, X. F. Sun, S. Ono, Y. Abe, S. Komiya, K. Segawa and Y. Ando: Phys. Rev. Lett. 96 (2006) 017008.

63) For a recent review, I. S. Beloborodov, A. L. Lopatin, V. M. Vinokur and K. B. Efetov: cond-mat/0603522.

64) A. V. Mahajan, H. Alloul, G. Collin and J.-F. Marucco: Phys. Rev. Lett. 72 (1994) 3100.

65) M.-H. Julien, T. Fehér, M. Horvatić, C. Berthier, O. N. Bakharev, P. Ségransan, G. Collin and J.-F. Marucco: Phys. Rev. Lett. 84 (2000) 3422.

66) W. A. MacFarlane, J. Bobroff, H. Alloul, P. Mendels, N. Blanchard, G. Collin and J.-F. Marucco: Phys. Rev. Lett. 85 (2000) 1108.

67) Y. Itoh, T. Machi, C. Kasai, S. Adachi, N. Watanabe, N. Koshizuka and M. Murakami: Phys. Rev. B 67 (2003) 064516.

68) Y. Sidis, P. Bourges, B. Hennion, L. P. Regnault, R. Villeneuve, G. Collin and J. F. Marucco: Phys. Rev. B 53 (1996) 6811.

69) M. Kofu, H. Kimura and K. Hirota: Phys. Rev. B 72 (2005) 064502.

70) J. E. Hoffmann, K. McElroy, D.-H. Lee, K. M. Lang, H. Eisaki, S. Uchida and J. C. Davis: Science 297 (2002) 1148; K. McElroy, R. W. Simmonds, J. E. Hoffman, D. H. Lee, J. Orenstein, H. Eisaki, S. Uchida, J. C. Davis: Nature 422 (2003) 592; Q.-H. Wang and D.-H. Lee: Phys. Rev. B 67 (2003) 020511(R).

71) The impurity effect near the anti-ferromagnetic critical point has been investigated in N. Bulut: Phys. Rev. B 61 (2000) 9051; Y. Ohashi: J. Phys. Soc. Jpn. 70 (2001) 2054; D. Matsunaka, H. Kasai, W. A. Dino and H. Nakanishi: Phys. Rev. B 68 (2003) 054503. H. Kontani and M. Ohno: Phys. Rev. B 74 (2006) 014406. See also, H. Tsuchiura, Y. Tanaka, M. Ogata and S. Kashiwaya: Phys. Rev. B 64 (2001) 140501.

72) S. Oauzi, J. Bobroff, H. Alloul and W. A. MacFarlane, Phys. Rev. B 70 (2004) 104515 and references there in.

73) Y. Itoh, T. Machi, N. Watanabe, S. Adachi, C. Kasai, N. Koshizuka: Physica C 378-381 (2002) 203.

74) M. Vershinin, S. Misra, S. Ono, Y. Abe, Y. Ando, A. Yazdani: 
Science 303 (2004) 1995.

75) T. Shibauchi and S. Horiuchi, in proceedings of $\mathrm{M}^{2} \mathrm{~S}-\mathrm{HTSC}$ VIII (to be published in Physica C).

76) Y. J. Uemura: Phys. Rev. Lett. 62 (1989) 2317.

77) Z. A. Xu, N. P. Ong, Y. Wang, T. Kakeshita and S. Uchida: Nature 406 (2000) 486; Y. Wang, Z. A. Xu, T. Kakeshita, S. Uchida, S. Ono, Y. Ando and N. P. Ong: Phys. Rev. Lett. 88 (2002) 257003.

78) J. Corson, R. Mallozzi, J. Orenstein, J. N. Eckstein and I. Bozovic: Nature 398 (1999) 221.

79) H. Mukuda, M. Abe, Y. Araki, Y. Kitaoka, K. Tokiwa, T. Watanabe, A. Iyo, H. Kito and Y. Tanaka: Phys. Rev. Lett. 96 (2006) 087001; H. Mukuda, M. Abe, S. Shimizu, Y. Kitaoka, A. Iyo, Y. Kodama, H. Kito, Y. Tanaka, K. Tokiwa and T. Watanabe: cond-mat/0610133.

80) K. Kanoda, Hyperfine Interactions 104 (1997) 235.

81) T. Jujo and K. Yamada: J. Phys. Soc. Jpn. 68 (1999) 2198.

82) G.-q. Zheng, T. Sato, Y. Kitaoka, M. Fujita, K. Yamada, Phys. Rev. Lett. 90 (2003) 197005.

83) K. Yamada, K. Kurahashi, T. Uefuji, M. Fujita, S. Park, S.-H. Lee and Y. Endoh, Phys. Rev. Lett. 90 (2003) 137004.

84) N. P. Armitage, D. H. Lu, C. Kim, A. Damascelli, K. M. Shen, F. Ronning, D. L. Feng, P. Bogdanov, Z.-X. Shen, Y. Onose, Y.
Taguchi, Y. Tokura, P. K. Mang, N. Kaneko, and M. Greven, Phys. Rev. Lett. 87 (2001) 147003; N. P. Armitage, F. Ronning, D. H. Lu, C. Kim, A. Damascelli, K. M. Shen, D. L. Feng, H. Eisaki, Z.-X. Shen, P. K. Mang, N. Kaneko, and M. Greven, Y. Onose, Y. Taguchi and Y. Tokura, Phys. Rev. Lett. 88 (2002) 257001; H. Matsui, K. Terashima, T. Sato, T. Takahashi, S.-C. Wang, H.-B. Yang, H. Ding, T. Uefuji and K. Yamada: Phys. Rev. Lett. 94 (2005) 047005.

85) M. Fujita, T. Kubo, S. Kuroshima, T. Uefuji, K. Kawashima, K. Yamada, I. Watanabe, and K. Nagamine: Phys. Rev. B 67 (2003) 014514.

86) Y. Kitaoka et al.: J. Phys. Soc. Jpn. 74 (2005) 186.

87 ) For a microscopic theory on the $s$-wave superconductivity, $\mathrm{N}$. Trivedi, R. T. Scalettar and M. Randeria: Phys. Rev. B 54 (1996) R3756.

88) An importance of the competing order and the similarity to the manganaites have been pointed out in E. Dagotto: condmat/0509041. See also, G. Alvarez, M. Mayr, A. Moreo and E. Dagotto: Phys. Rev. B 71 (2005) 014514; M. Mayr, G. Alvarez, A. Moreo and E. Dagotto: Phys. Rev. B 73 (2006) 014509.

89) For example, the phenomenological XY model has been derived in the disordered $s$-wave superconductor. A. Ghosal, M. Randeria and N. Trivedi: Phys. Rev. B 65 (2002) 014501. 